\title{
Fear of cancer recurrence among cancer survivors in Singapore
}

\author{
Rathi Mahendran ${ }^{1}$, MMed, Jianlin Li $\underline{i u}^{1,2}$, PhD, Sangita Kuparasundram ${ }^{3}$, MRCP, MMed, Sebastian Simard ${ }^{4}$, PhD, \\ Yiong Huak Chan ${ }^{5}$, PhD, Ee Heok Kua ${ }^{1}$, MD, Konstadina Griva ${ }^{6}$, PhD
}

INTRODUCTION Fear of cancer recurrence (FCR) among cancer survivors is a persistent and distressing psychosocial concern that affects recovery and quality of life. The prevalence of FCR in Singapore is unknown. This cross-sectional study was designed to examine FCR and identify factors associated with FCR in mixed-cancer survivors locally. METHODS Cancer survivors in remission $(n=404)$ were assessed for: FCR using the Fear of Cancer Recurrence Inventory (FCRI); emotional distress using the Hospital Anxiety and Depression Scale; and quality of life using the World Health Organization Quality of Life-BREF. Clinical and severe/pathological FCR was determined based on the severity scale of FCRI, known as FCRI-Short Form. Multivariate logistic regression was performed to examine factors associated with FCR. RESULTS The mean score on the FCRI was $59.5 \pm 30.4$. 43.6\% of cancer survivors had clinical FCR and $32.1 \%$ had severe/pathological FCR. Younger age (odds ratio [OR] 0.952, 95\% confidence interval [CI] 0.911-0.995, p < 0.05), higher educational status (OR 2.55, 95\% Cl 1.15-5.65, $\mathrm{p}<0.05)$ and higher levels of emotional distress (OR 1.17, 95\% Cl 1.10-1.24, $\mathrm{p}<0.001$ ) were significantly associated with severe/pathological levels of FCR.

CONCLUSION The present study is the first to determine levels of FCR among cancer survivors in Singapore. While the total FCR scores were similar to those of international studies, severe/pathological levels of FCR were found to be four times higher. These findings highlight a problem that is not widely recognised or acknowledged, but which deserves greater attention.

Keywords: cancer survivors in Singapore, fear of cancer recurrence

\section{INTRODUCTION}

The diagnosis of cancer can be devastating for many patients regardless of the stage of cancer, available treatment and prognosis. ${ }^{(1)}$ Equally debilitating is the fear of cancer recurrence (FCR), which occurs across the continuum of the illness and even after successful treatment. FCR has been endorsed as one of the top five greatest concerns of cancer survivors and described as a 'universal concern' across cancer populations. ${ }^{(2-4)}$ Persistent, elevated FCR is known to be associated with clinical depression, anxiety, post-traumatic stress symptoms and poor quality of life among cancer survivors. ${ }^{(3,5)}$ When it occurs in the survivorship phase, the fear is comparable between short- and long-term survivors, suggesting 'persistence throughout survivorship'. McGinty et al have conceptualised this fear as multidimensional with cognitive, affective and behavioural components, ${ }^{(6)}$ and a cognitive behavioural model has been proposed as a theoretical framework for therapy. ${ }^{(7-9)} \mathrm{A}$ recent colloquium of experts in the field of FCR has established the following relevant definition of FCR for research and clinical use: "fear, worry, or concern about cancer returning or progressing" ${ }^{\prime(10)}$

Research on the prevalence and severity of FCR among cancer survivors has mainly been conducted in Western populations. These have reported a wide range of 39\%-97\% (average $73 \%$ ) of survivors having some degree of FCR. ${ }^{(11)}$ About $27 \%-87 \%$ (average $49 \%$ ) of these cancer survivors reported moderate to high degrees of FCR, while approximately $0 \%-15 \%$ (average $7 \%$ ) of cancer survivors reported high levels of FCR. ${ }^{(2,11)}$ The variability of the results may be attributable, to some extent, to the different measures used to assess FCR. ${ }^{(12)}$ There are several brief scales that have been used to measure FCR, and they range from two to five items (Table I). ${ }^{(13-22)}$ However, measures that rely on a single item or focus on limited dimensions, such as severity, frequency or functional consequences, may not adequately capture the multidimensionality of FCR and thus, limit their overall validity and reliability. ${ }^{(11)}$ There are four longer scales that measure FCR (Table I). ${ }^{(23-26)}$ Of these, the Fear of Cancer Recurrence Inventory (FCRI) was developed to measure several aspects of FCR, reflecting the multidimensional nature of FCR. ${ }^{(26)}$

Gaps in understanding of FCR have resulted from contradictory findings of the key factors associated with FCR. ${ }^{(2)}$ A 2013 systematic review of 43 studies by Crist and Grunfeld found that younger age most consistently predicted elevated FCR, while other factors were reported in some but not all studies, including higher levels of emotional distress and lower quality of life. ${ }^{(5)}$ Importantly, this review highlighted that the majority of studies were focused on one type of cancer, specifically breast or gynaecological cancers, with a lack of research on mixed-cancer populations. ${ }^{(5)}$ Furthermore, research to date has been predominantly conducted in Western populations, with only two studies ${ }^{(27,28)}$ from the abovementioned systematic review from Asian countries: the Japanese study used a single item to measure FCR, ${ }^{(27)}$ while the South Korean study used the Fear of Progression Questionnaire. ${ }^{(28)}$

\footnotetext{
${ }^{1}$ Department of Psychological Medicine, Yong Loo Lin School of Medicine, National University of Singapore, ${ }^{2}$ Research Department, Institute of Mental Health, ${ }^{3}$ Ministry of Health Holdings, Singapore, ${ }^{4}$ Health Science Department, Université du Quebec a Chicoutimi (UQAC), Quebec, Canada, ${ }^{5}$ Biostatistics Unit, Yong Loo Lin School of Medicine, National University of Singapore, ${ }^{6}$ Department of Health Psychology and Behavioural Medicine, Lee Kong Chian School of Medicine, Nanyang Technological University, Singapore Correspondence: A/Prof Rathi Mahendran, Senior Consultant Psychiatrist and Associate Professor, Department of Psychological Medicine, Yong Loo Lin School of Medicine, National University of Singapore, NUHS Tower Block, Level 9, 1E Kent Ridge Road, Singapore 119228. medrm@nus.edu.sg
} 
Table I. Scales for the measurement of fear of cancer recurrence. ${ }^{(12)}$

\begin{tabular}{|c|c|c|}
\hline Scale for fear of cancer recurrence measurement & No. of items & Study, year \\
\hline \multicolumn{3}{|l|}{ Brief scales } \\
\hline Lasry \& Margolese Fear of Recurrence Index (LMRI) & 2 & Lasry \& Margolese, ${ }^{(13)} 1992$ \\
\hline Cancer Worry Scale (B) (CWS-B) & 2 & Cameron et al, ${ }^{(14)} 2007$ \\
\hline Worry about Prostate Cancer Scale (WPCS) & 2 & Diefenbach et al, ${ }^{(15)} 2008$ \\
\hline Worry of Cancer Scale Revised (WOC-R) & 2 & Hodges \& Humphris, ${ }^{(16)} 2009$ \\
\hline Cancer Worry Scale-A (CWS-A) & 3 & Easterling \& Leventhal, ${ }^{(17)} 1989$ \\
\hline Fear of Recurrence Scale (B) (FRSb) & 3 & Franssen et al, ${ }^{(18)} 2009$ \\
\hline Fear of Recurrence Scale (A) (FRSa) & 4 & Rabin et al, ${ }^{(19)} 2004$ \\
\hline Cancer-related Worries Scale (CRWS) & 4 & Deimling et al, ${ }^{(20)} 2006$ \\
\hline Fear of Relapse/Recurrence Scale (FRRS) & 5 & Greenberg et al, ${ }^{(21)} 1997$ \\
\hline Assessment of Survivor Concerns Scale (ASCS) & 5 & Gotay \& Pagano, (22) 2007 \\
\hline \multicolumn{3}{|l|}{ Longer scales } \\
\hline Fear of Recurrence Questionnaire (FRQ) & 22 & Northouse, ${ }^{(23)} 1981$ \\
\hline Fear of Progression Questionnaire (FOP-Q) & 43 & Herschbach et al, ${ }^{(24)} 2005$ \\
\hline Concerns About Recurrence Scale (breast cancer specific) & 30 & Vickberg, ${ }^{(25)} 2003$ \\
\hline Fear of Cancer Recurrence Inventory (FCRI) & 42 & Simard \& Savard, (26) 2009 \\
\hline
\end{tabular}

Therefore, applicability to non-Western populations is unknown. Recommendations have been made for further research to better understand the impact of sociodemographic variables (e.g. gender) and medical variables on FCR in mixed-cancer populations. ${ }^{(5)}$ Additionally, research in our local setting would provide a clearer profile of cancer survivors in Singapore, help us identify those at risk and understand their needs, and enable us to determine the factors associated with FCR for service development and delivery.

The aim of this study was to examine the prevalence of FCR and identify the sociodemographic and psychological factors associated with FCR among mixed-cancer survivors in Singapore. No a priori hypothesis was made, as there have been no previous studies in the local population.

\section{METHODS}

A total of 927 participants were identified and approached during their follow-up visits at the National University Cancer Institute, Singapore. 462 participants were recruited from February 2015 to June 2016. Inclusion criteria were: (a) a diagnosis of cancer; (b) at least one year since the completion of treatment (surgery, chemotherapy and/or radiotherapy); (c) cancer status in remission; (d) age 21-84 years old; (e) Singapore citizens or permanent residents; and ( $\mathrm{f}$ ) ability to understand and read English or Mandarin. Ethics approval was granted by the National Healthcare Group Domain Specific Review Board A (reference number: 2015/00003). All participants provided written informed consent prior to being included in the study.

This was a cross-sectional study that involved a questionnaire methodology. Participants completed a self-report questionnaire on sociodemographic characteristics (e.g. gender, age, ethnicity, marital status, education and occupation) and medical variables (e.g. comorbid chronic physical conditions, cancer type and stage, type of cancer treatment received and time since completion of treatment).
FCR was measured with the FCRI, a 42-item self-report questionnaire that provides a multidimensional assessment of FCR. The FCRI has been found to be suitable for mixedcancer populations who differ in diagnoses and time since diagnosis. ${ }^{(26)}$ The questionnaire evaluates seven components: the presence of potential stimuli activating FCR; the presence and severity of intrusive thoughts or images associated with FCR; the potential consequences of FCR (both psychological distress and reassurance behaviours); the level of self-criticism towards FCR intensity; and the coping strategies that can be used to cope with FCR. ${ }^{26)}$ The FCRI was initially developed in French, and the English version was validated in a group of mixed cancer patients in Canada. ${ }^{(29)}$ The English and Mandarin versions of the FCRI have been validated in the local cancer population, and both measures were observed to have good psychometric properties. ${ }^{(30)}$ Participants rate the items on a Likert scale from 0 (not at all or never) to 4 (a great deal or all the time). Only one item (Item 13) is reverse-scored, and total scores are obtained for each subscale and for the entire scale by summing up the items; higher scores indicate higher levels of FCR. ${ }^{(26)}$

The severity subscale of the FCRI forms the nine-item FCRIShort Form (FCRI-SF); it has strong correlations with the total FCRI score and is used to determine severe/pathological levels of FCR. ${ }^{(26)}$ A cut-off score of 13 or higher on the FCRI-SF is associated with optimal sensitivity (88\%) and specificity (75\%) for screening clinical levels of FCR. ${ }^{(31)}$ Research has also suggested a higher cutoff score of 16 (increases specificity to $97 \%$ but lowers sensitivity) as an optimal diagnostic cut-off for severe/pathological levels of FCR. ${ }^{(31)}$ The present study used the FCRI-SF cut-off score of 13 to establish clinical levels of FCR and the cut-off score of 16 to determine the prevalence of severe/pathological levels of FCR.

The 14-item questionnaire, Hospital Anxiety and Depression Scale (HADS) was used to measure the severity of patients' emotional distress. The HADS, a validated self-report measure designed for use in a hospital setting, comprises two subscales 
Table II. Sociodemographic and clinical characteristics of participants $(n=404)$.

\begin{tabular}{|c|c|c|c|}
\hline Variable & Mean \pm SD/no. $(\%)^{*}$ & Variable & Mean \pm SD/no. (\%)* \\
\hline Age (yr) & $55.8 \pm 11.5$ & Cancer stage & \\
\hline Gender & & Early (Stage 1 or 2 ) & $226(70)$ \\
\hline Male & $80(20)$ & Late (Stage 3 or 4 ) & $98(30)$ \\
\hline Female & $324(80)$ & Treatment completed & \\
\hline Marital status & & Chemotherapy & $229(58)$ \\
\hline Married & $112(28)$ & Radiotherapy & $117(45)$ \\
\hline Divorced/single/others & $287(72)$ & Surgery & $292(74)$ \\
\hline Ethnicity & & Time since treatment completion (mth) & $63.2 \pm 62.1$ \\
\hline Chinese & $318(80)$ & Chronic physical comorbidities $^{\dagger}$ & \\
\hline Malay & $44(11)$ & Yes & $188(80)$ \\
\hline Indian & $25(6)$ & No & $47(20)$ \\
\hline Others & $14(4)$ & FCR & $173(44)$ \\
\hline Education & & Severe/pathological FCR & $128(32)$ \\
\hline Secondary and below & $271(68)$ & FCR dimensions & \\
\hline Tertiary and above & $128(32)$ & Triggers & $12.9 \pm 7.2$ \\
\hline Occupational status & & Severity & $12.3 \pm 6.8$ \\
\hline Employed & $219(56)$ & Psychological distress & $4.6 \pm 4.3$ \\
\hline Unemployed & $173(44)$ & Functional impairment & $6.8 \pm 6.7$ \\
\hline Cancer type & & Insight & $2.3 \pm 2.7$ \\
\hline Breast & $154(39)$ & Reassurance & $3.8 \pm 3.2$ \\
\hline Gynaecologic & $106(27)$ & Coping strategies & $16.8 \pm 9.4$ \\
\hline Colorectal & $55(14)$ & Emotional distress & $8.3 \pm 6.6$ \\
\hline Multisite & $16(4)$ & Quality of life domains & \\
\hline Nasopharyngeal & $14(4)$ & Physical & $60.5 \pm 19.2$ \\
\hline Haematological & $14(4)$ & Psychological & $62.9 \pm 17.5$ \\
\hline Lung & $14(4)$ & Social relations & $58.8 \pm 21.0$ \\
\hline Pancreas & $3(1)$ & Environmental & $55.6 \pm 20.4$ \\
\hline Brain tumour & $2(1)$ & & \\
\hline
\end{tabular}

*Percentage values are used as valid percentages (of participants) and not exact percentages of total sample. †Chronic physical comorbidities (yes/no) refer to at least one diagnosis of hypertension, hyperlipidaemia, diabetes mellitus, stroke, renal disease, cardiac conditions or arthritis. FCR: fear of cancer recurrence; SD: standard deviation

measuring depression and anxiety. ${ }^{(32)}$ Previous research has validated the use of the HADS in the Singapore cancer population. ${ }^{(33)}$ The subscales in the HADS may be combined to obtain a total distress score (ranging from 0 to 42) as a measure of emotional distress. Participants were asked to rate how they felt in the past week on a 4-point Likert scale $(0=$ not at all to $3=$ most of the time). Higher scores obtained on the HADS indicate greater emotional distress. ${ }^{(32)}$ Scores $<7$ indicate non-cases. ${ }^{(34)}$

The 26-item World Health Organization Quality of Life Instrument-Short Version (WHOQOL-BREF), an abbreviated version of the WHOQOL, was used to measure four domains of quality of life (physical, psychological, social relations and environmental). Participants were asked to score how they felt about different aspects of their life in the past four weeks on a 5-point Likert scale ( $1=$ not at all to $5=$ an extreme amount). Higher scores indicate a better self-perceived quality of life in each domain. ${ }^{(35)}$

Descriptive and regression analyses were performed using IBM SPSS Statistics version 23.0 (IBM Corp, Armonk, NY, USA). Mean and standard deviation were calculated for continuous variables, and frequencies and percentages for all other categorical variables. Pearson's correlation was conducted to examine the associations between overall FCR, emotional distress and quality of life domains (physical, psychological, social relations and environmental). Multivariate logistic regression analysis was performed to examine the sociodemographic (gender, age, ethnicity, marital status, education, occupation), medical (comorbid chronic physical conditions, cancer type and stage, type of cancer treatment received and time since completion of treatment), and psychological (emotional distress and quality of life domains) variables that were associated with severe/pathological $(\geq 16)$ levels of FCR.

\section{RESULTS}

Completed data was available for 404 participants. Table II presents the participants' sociodemographic and medical characteristics. Notably, participants were mostly female $(80 \%)$ with early stage cancer (70\%).

The mean emotional distress score was $8.3 \pm 6.6$ (Table II); the scores on the HADS range were mild: 8-10, moderate: 11-14 and severe: $15-21$. Self-perceived quality of life was the lowest 
Table III. Associations between fear of cancer recurrence (FCR), emotional distress, and quality of life domains.

\begin{tabular}{|lllllll|}
\hline Pearson's r & FCR & Emotional distress & Physical & Psychological & Social relations & Environmental \\
\hline FCR & 1 & & & & & \\
\hline Emotional distress & $0.61^{*}$ & 1 & & & & \\
\hline Physical & $-0.22^{*}$ & $-0.53^{*}$ & $0.70^{*}$ & 1 & 1 & 1 \\
\hline Psychological & $-0.31^{*}$ & $-0.65^{*}$ & $0.54^{*}$ & $0.63^{*}$ & $0.63^{*}$ & 1 \\
\hline Social relations & $-0.19^{*}$ & $-0.39^{*}$ & $0.65^{*}$ & $0.74^{*}$ & \\
\hline Environmental & $-0.18^{*}$ & $-0.44^{*}$ & & & & \\
\hline
\end{tabular}

${ }^{*}$ Correlation significant at $\mathrm{p}<0.01$.

Table IV. Results from logistic regression model examining the sociodemographic, medical, and psychological correlates of overal and severe/pathological fear of cancer recurrence.

\begin{tabular}{|llll|}
\hline \multirow{2}{*}{ Variable } & \multicolumn{3}{c|}{ Logistic regression model* } \\
\cline { 2 - 4 } & Odds ratio & $\mathbf{9 5 \%} \mathbf{C l}$ & p-value \\
\hline Age & 0.952 & $0.911-0.995$ & $<0.05$ \\
\hline Emotional distress & 1.17 & $1.10-1.24$ & $<0.001$ \\
\hline $\begin{array}{l}\text { Education (tertiary and } \\
\text { above) }\end{array}$ & 2.55 & $1.15-5.65$ & $<0.05$ \\
\hline $\begin{array}{l}\text { Education (secondary } \\
\text { and below) }\end{array}$ & Reference & & \\
\hline
\end{tabular}

*Logistic regression model included age, gender, ethnicity, education status, occupation status, cancer stage, cancer type, type of treatment completed time since treatment completion, chronic physical comorbidities, emotional distress and quality of life domains (physical, psychological, social relations and environmental). However, only variables with significant associations with fear of cancer recurrence were detailed above. $\mathrm{Cl}$ : confidence interval

on the environmental domain of the WHOQOL-BREF (which included eight items: financial resources; accessibility and quality of health and social care, home and physical environment; and participation and opportunities for recreation/leisure activities). It was the highest on the psychological domain (which included six items: bodily image and appearance; negative and positive feelings; self-esteem; spirituality and cognition). The mean FCRI score was $59.5 \pm 30.4$. On the FCRI-SF, $43.6 \%$ of participants had clinical FCR (cut-off score $\geq 13$ ) and $32.1 \%$ experienced severe/ pathological levels of FCR (cut-off score $\geq 16$ ).

Table III presents the correlations between overall FCR, emotional distress and quality of life domains. FCR was positively associated with the emotional distress domain of quality of life $(r=0.61, p<0.01)$ and negatively associated with the physical $(r=-0.22, p<0.01)$, psychological $(r=-0.31, p<0.01)$, social relations $(r=-0.19, p<0.01)$ and environmental $(r=-0.18$, $\mathrm{p}<0.01$ ) domains. Emotional distress was also negatively associated with the same quality of life domains.

Table IV presents the results of the multivariate logistic regression analysis, which examined the sociodemographicmedical and psychological correlates of severe/pathological FCR. Firstly, multivariate logistic regression analysis revealed that a one-year age increment had at least a $5 \%$ reduction in risk of severe/pathological levels of FCR (odds ratio [OR] 0.952, $95 \%$ confidence interval $[\mathrm{Cl}] 0.911-0.995, \mathrm{p}<0.05)$. Secondly, it showed that the odds of experiencing severe/pathological FCR for those with a higher educational status (tertiary and above) was 2.55 times $(95 \% \mathrm{Cl} 1.15-5.65, \mathrm{p}<0.05)$ that of those with a lower educational status (secondary and below). Thirdly, it revealed that higher levels of emotional distress (OR 1.17, 95\% Cl $1.10-1.24, p<0.001)$ were significantly associated with severe/ pathological levels of FCR.

\section{DISCUSSION}

The present study is the first to examine the prevalence, severity and factors associated with FCR among mixed cancer survivors in Singapore. The level of FCR (mean $59.5 \pm 30.4$ ) was comparable to that of two other studies (mean $51.7 \pm 28.8$ and $53.8 \pm 27.8$, respectively) conducted in Western mixed-cancer populations, ${ }^{(4,26)}$ suggesting similarities across cultures. While the prevalence of clinical FCR in our study was similar to that of Western populations (43.6\% vs. 46\%), severe/pathological FCR among cancer survivors in Singapore was slightly more than four times that reported in Western populations $(32.1 \%$ vs. $7 \%){ }^{(2)}$ Several issues need to be considered when interpreting these findings. Clinical assessments were not included in our study design and participants who reported levels of clinical FCR were not assessed by a clinician, which would have provided a definitive diagnosis. Additionally, there is still no consensus on the distinctions between mild, moderate and severe levels of FCR. ${ }^{(36)}$ Despite these limitations, our findings suggest that clinicians should be concerned about and aware of FCR. Screening, assessments and patient and caregiver psycho-education should be priority measures.

We have noted that among cancer survivors in Singapore, younger age, higher educational status and higher level of emotional distress are significantly associated with overall and severe/pathological FCR. Crist and Grunfeld have suggested that younger cancer survivors might have more difficulties with managing the uncertainties associated with cancer recurrence. ${ }^{(5)}$ This has been attributed to greater financial commitments faced by younger adults, who may be at an early stage in their career, with lower income, little savings and greater family responsibilities. Shim et al, examining concerns related to cancer progression among Korean cancer patients, found that familial concerns, specifically a perceived sense of being a burden to one's family, was the most predominant concern. ${ }^{(28)}$ An earlier qualitative study conducted in cancer caregivers in Singapore supports the notion of burden in the patient-caregiver interaction. The study revealed that cancer caregivers provide care because of family obligations and filial piety, that family caregiving is not uniformly experienced as positive, and that it is burdensome for those providing care for extrinsic motivations. ${ }^{(37)}$ The role and impact of familial concerns 
and motivations on a cancer patient may be a unique aspect of Asian cultures, and necessitates further exploration among cancer survivors in Singapore.

There is considerable evidence in the literature to account for the relationship between emotional distress and FCR, such as higher levels of catastrophic thinking and worry that may result in greater FCR. ${ }^{(28,38,39)}$ While the present study did not determine the cause of emotional distress symptoms, the findings underscore the importance of continued psychological care during the post-treatment phase. Untreated emotional distress due to other concerns (e.g. body image concerns following completion of treatment) ${ }^{(40)}$ may later serve as a risk factor for FCR. ${ }^{(25)}$

The association between higher educational status and severe/ pathological levels of FCR could be due to these individuals having knowledge, resources and the ability to seek more information about their illness, treatment and prognosis. A greater awareness of risk factors and the implications of cancer recurrence may have also resulted in more catastrophic thinking, leading to increased severity of FCR.

Unlike previous studies, ${ }^{(5)}$ the present study did not find an association between quality of life domains (physical, psychological, social relations and environmental) and FCR. However, our earlier research has shown that specific psychological resources (e.g. mindfulness and spirituality) have a role in alleviating emotional distress among Singapore cancer patients. ${ }^{(41)}$ The possibility of using these resources and enhancing them in cancer survivors may potentially be protective against FCR.

There were no significant results for gender and ethnicity, both of which have been reported to be associated with more contradictory findings in the FCR literature. ${ }^{(5)}$ Finally, similar to the majority of research studies in the FCR literature, ${ }^{(5)}$ the present study did not observe significant associations between medical variables (e.g. cancer and treatment-related factors) and FCR. This may suggest that underlying cognitive processes may be more important in FCR, affecting all survivors across a broad spectrum of cancer types, medical comorbidities and treatment modalities.

There are several potential limitations to the present study, the most important being the cross-sectional study design, which precludes any causal inferences. Longitudinal research is required to examine the temporal effects of predictors and changes in FCR over time. Secondly, selection bias should be considered as a possible reason for the high levels of severe/pathological levels of FCR. Distressed patients may have agreed to participate, while those who were not distressed or were experiencing mild levels of distress may not have participated. Thirdly, the use of self-report measures may introduce response bias. Fourthly, a mixed-cancer sample may conflate a range of cancer severity, and factors associated with specific cancer types may not be elucidated in this study. However, examining FCR in a mixed-cancer sample remains important, as this is in line with the international call for additional research in this area. ${ }^{(2,5)}$ Importantly, findings based on a mixed-cancer sample may be generalised to a wider, heterogeneous population of cancer survivors in Singapore. Lastly, while the FCRI may have been recently validated in the local population, ${ }^{(16)}$ caution must be taken to interpret the prevalence rates of severe/pathological FCR based on the cut-offs established in Western populations.

Notwithstanding these limitations, the present study contributes new knowledge pertaining to the level of FCR and prevalence of severe/pathological FCR in mixed-cancer survivors in Singapore. Importantly, the present study has also supported and clarified previous findings on factors associated with elevated and clinical levels of FCR. The use of a large sample size $(n=404)$ addresses the methodological limitations in previous small studies, ${ }^{(2,5)}$ but also suggests the need for larger epidemiologicaltype studies to examine the problem locally.

The study results would be useful to clinicians and therapists in dealing with patient's concerns about their illness and disease status, designing relevant post-treatment interventions and, ultimately, enhancing psychosocial care of cancer survivors. In service delivery, prioritising the needs of cancer survivors with severe/pathological levels of FCR is a primary concern and screening programmes need to be developed. Further research in a longitudinal study of risk factors and protective factors will support this initiative, as well as address the lack of consensus on the different levels of FCR severity.

\section{ACKNOWLEDGEMENTS}

We acknowledge Dr S Simard for permission to use the Fear of Cancer Recurrence Inventory, which he developed. This research was supported by the Singapore Cancer Society, Cancer Research Grant 2014 (February 2015 to January 2017). The funding body had no involvement in the study design, collection, analysis or interpretation of data, writing of the manuscript and the decision to submit the manuscript for publication. The authors declare no conflict of interests.

\section{REFERENCES}

1. Moser RP, Arndt J, Han PK, et al. Perceptions of cancer as a death sentence: prevalence and consequences. J Health Psychol 2014; 19:1518-24.

2. Simard S, Thewes B, Humphris G, et al. Fear of cancer recurrence in adult cancer survivors: a systematic review of quantitative studies. J Cancer Surviv $2013 ; 7: 300-22$.

3. Koch $L$, Jansen $L$, Brenner $H$, Arndt V. Fear of recurrence and disease progression in long-term ( $\geq 5$ years) cancer survivors--a systematic review of quantitative studies. Psychooncology 2013; 22:1-11.

4. Simard S, Savard J, Ivers H. Fear of cancer recurrence: specific profiles and nature of intrusive thoughts. J Cancer Surviv 2010; 3:361-71.

5. Crist JV, Grunfeld EA. Factors reported to influence fear of recurrence in cancer patients: a systematic review. Psychooncology 2013; 22:978-86.

6. McGinty HL, Small BJ, Laringa C, Jacobsen PB. Predictors and patterns of fear of cancer recurrence in breast cancer survivors. Health Psychol 2016; 35:1-9.

7. Cohee AA, Adams RN, Johns SA, et al. Long-term fear of recurrence in young breast cancer survivors and partners. Psychooncology 2017; 26:22-8

8. Fardell JE, Thewes B, Turner J, et al. Fear of cancer recurrence: a theoretical review and novel cognitive processing formulation. J Cancer Surviv 2016; 10:663-73

9. Lee-Jones C, Humphris G, Dixon R, Hatcher MB. Fear of cancer recurrence--a literature review and proposed cognitive formulation to explain exacerbation of recurrence fears. Psychooncology 1997; 6:95-105.

10. Lebel S, Ozakinci G, Humphris G, et al. From normal response to clinical problem: definition and clinical features of fear of cancer recurrence. Support Care Cancer 2016; 24:3265-8

11. Thewes $B$, Butow $P, Z$ Zachariae $R$, et al. Fear of cancer recurrence: a systematic literature review of self-report measures. Psychooncology 2012; 21:571-87.

12. Cancer Australia. Recommendations for the identification and management of fear of cancer recurrence in adult cancer survivors. Appendix A: Summary of validated tools to assess FCR. Available at: https://guidelines.canceraustralia.gov. au/guidelines/fear_of_recurrence/ch01s17.php. Accessed February 22, 2019 
13. Lasry JC, Margolese RG. Fear of recurrence, breast-conserving surgery, and the trade-off hypothesis. Cancer 1992; 69:2111-5.

14. Cameron LD, Booth RJ, Schlatter $M$, et al. Changes in emotion regulation and psychological adjustment following use of a group psychosocial support program for women recently diagnosed with breast cancer. Psychooncology 2007; 16:171-80.

15. Diefenbach M, Mohamed NE, Horwitz E, Pollack A. Longitudinal associations among quality of life and its predictors in patients treated for prostate cancer: the moderating role of age. Psychol Health Med 2008; 13:146-61.

16. Hodges LJ, Humphris GM. Fear of recurrence and psychological distress in head and neck cancer patients and their carers. Psychooncology 2009; 18:841-8.

17. Easterling DV, Leventhal $\mathrm{H}$. Contribution of concrete cognition to emotion: neutral symptoms as elicitors of worry about cancer. J Appl Psychol 1989; 74:787-96.

18. Franssen SJ, Lagarde SM, van Werven JR, et al. Psychological factors and preferences for communicating prognosis in esophageal cancer patients. Psychooncology 2009; 18:1199-207.

19. Rabin C, Leventhal H, Goodin S. Conceptualization of disease timeline predicts posttreatment distress in breast cancer patients. Health Psychol 2004; 23:407-12.

20. Deimling GT, Wagner LJ, Bowman KF, et al. Coping among older-adult, long-term cancer survivors. Psychooncology 2006; 15:143-59.

21. Greenberg DB, Kornblith AB, Herndon JE, et al. Quality of life for adult leukemia survivors treated on clinical trials of Cancer and Leukemia Group B during the period 1971-1988: predictors for later psychologic distress. Cancer 1997; 80:1936-44

22. Gotay CC, Pagano IS. Assessment of Survivor Concerns (ASC): a newly proposed brief questionnaire. Health Qual Life Outcomes 2007; 5:15.

23. Northouse LL. Mastectomy patients and the fear of cancer recurrence. Cancer Nurs $1981 ; 4: 213-20$

24. Herschbach P, Berg P, Dankert A, et al. Fear of progression in chronic diseases: psychometric properties of the Fear of Progression Questionnaire. J Psychosom Res 2005; 58:505-11.

25. Vickberg SM. The Concerns About Recurrence Scale (CARS): a systematic measure of women's fears about the possibily of breast cancer reccurrence. Ann Behav Med 2003; 25:16-24

26. Simard S, Savard J. Fear of Cancer Recurrence Inventory: development and initial validation of a multidimensional measure of fear of cancer recurrence. Support Care Cancer 2009; 17:241-51.

27. Noguchi $M$, Kitagawa $H$, Kinoshita $K$, et al. Psychologic and cosmetic selfassessments of breast conserving therapy compared with mastectomy and immediate breast reconstruction. J Surg Oncol 1993; 54:260-6.

28. Shim EJ, Shin YW, Oh DY, Hahm BJ. Increased fear of progression in cancer patients with recurrence. Gen Hosp Psychiatry 2010; 32:169-75

29. Lebel S, Simard S, Harris C, et al. Empirical validation of the English version of the Fear of Cancer Recurrence Inventory. Qual Life Res 2016; 25:311-21.

30. Liu J, Mahendran R, Chua SM, et al. Validation of the English and Mandarin versions of the Fear of Cancer Recurrence Inventory in an Asian population. J Health Psychol 2017; 1359105317727819.

31. Custers JAE, Gielissen MFM, Janssen SHV, de Wilt JHW, Prins JB. Fear of cancer recurrence in colorectal cancer survivors. Support Care Cancer 2016; 24:555-62.

32. Zigmond AS, Snaith RP. The hospital anxiety and depression scale. Acta Psychiatr Scand 1983; 67:361-70

33. Beck KR, Tan SM, Lum SS, Lim LE, Krishna LK. Validation of the emotion thermometers and hospital anxiety and depression scales in Singapore: screening cancer patients for distress, anxiety and depression. Asia Pac J Clin Oncol 2014; 12:e241-9.

34. Stern AF. The hospital anxiety and depression scale. Occup Med (Lond) 2014; 64:393-4.

35. World Health Organization. WHOQOL-BREF: Introduction, administration, scoring and generic version of the assessment: Field trial version. December 1996. Available at: https://apps.who.int/iris/handle/10665/63529. Accessed January 2, 2015.

36. Maheu C, Lebel S, Courbasson C, et al. Protocol of a randomized controlled trial of the fear of recurrence therapy (FORT) intervention for women with breast or gynecological cancer. BMC Cancer 2016; 16:291

37. Ng HY, Griva K, Lim HA, Tan JY, Mahendran R. The burden of filial piety: a qualitative study on caregiving motivations amongst family caregivers of patients with cancer in Singapore. Psychol Health 2016; 31:293-310.

38. Janz NK, Hawley ST, Mujahid MS, et al. Correlates of worry about recurrence in a multiethnic population-based sample of women with breast cancer. Cancer $2011 ; 117: 1827-36$.

39. Humphris GM, Rogers S, McNally D, et al. Fear of recurrence and possible cases of anxiety and depression in orofacial cancer patients. Int J Oral Maxillofac Surg 2003; 32:486-91

40. Liu J, Peh CX, Mahendran R. Body image and emotional distress in newly diagnosed cancer patients: the mediating role of dysfunctional attitudes and rumination. Body Image 2017; 20:58-64.

41. Lim HA, Griva K, Tan JY, Mahendran R. A longitudinal exploration of the psychological resources influencing depression and anxiety in newly diagnosed Asian persons with cancer. Psychooncology 2017; 26:278-81. 